An empirical approach of identifying subject matter experts (SMEs) for the development of situational judgment tests

\author{
Don C. Zhang ${ }^{1} \&$ Yi Wang ${ }^{2}$ \\ ${ }^{1}$ Louisiana State University \\ ${ }^{2}$ Pennsylvania State University
}

\begin{abstract}
Author Notes
Correspondence concerning this study should be sent to Don Zhang at zhang1@1su.edu.

Don C. Zhang, PhD, Department of Psychology, Louisiana State University, Baton Rouge, LA.

Yi Wang, PhD, Department of Psychology, Pennsylvania State University, State College, PA.

We declare no financial conflicts of interest in conducting or reporting this work.

\section{Version Notes}

Final draft of the manuscript accepted for publication in Journal of Personnel Psychology. This version is not the copy of record and may not exactly replicate the final, authoritative version of the article. Please cite this paper as: Zhang, D.C. \& Wang, Y. (in press, 2021). An empirical approach of identifying subject matter experts (SMEs) for the development of situational judgment tests. Journal of Personnel Psychology
\end{abstract}




\begin{abstract}
The development of a scoring key for the situational judgment test (SJT) often requires subject matter experts (SMEs) to identify the best responses for a hypothetical situation. And yet, there is no gold standard for identifying the SMEs. This paper describes an empirical and context-free approach: the Cochran-Weiss-Shanteau (CWS) method, which does not rely on external criteria such as tenure or credential. We first describe the theory behind the empirical approach of expertise. We also outline the CWS method and provide an $R$ script for calculating the CWSindex. Next, we demonstrate how the CWS-index can be used for improving interrater agreement and the efficiency of SME selection. Finally, we examined the nomological network of the CWS index. We found the CWS index was associated with reflective thinking and intuition avoidance. Keywords: situational judgment tests, subject matter expertise, test development, interrater agreement, rationality
\end{abstract}


An empirical approach of identifying subject matter experts (SMEs) for the development of situational judgment tests

Organizations regularly use situational judgment tests (SJT) in high-stakes preemployment testing situations due to their high predictive validity, low adverse impact, and resistance to faking (Christian et al., 2010; Lievens et al., 2008). In a typical SJT, the test taker reads about a job-related issue and indicates - from a set of possible choices - the best course of action to resolve the issue (Whetzel et al., 2008). SJT performance is assessed by the frequency to which the test taker chooses the correct response across the test items. As such, identifying the correct responses (scoring key) a priori is a vital step in the development of the SJT.

Test developers commonly consult the judgments of subject matter experts (SMEs) when developing the scoring key (the rational scoring method; see Bergman, Drasgow, Donovan, Henning, \& Juraska, 2006). A recent review of the literature showed that the rational scoring method is used in $64 \%$ of all SJTs: much more than any other method (Campion et al., 2014). Despite the prevalence of using SMEs for SJT development, the field has not agreed upon a gold standard for identifying who should be SMEs or the quality of their judgments. In theory, SMEs should be identified based on their job-related expertise, but in practice, expertise is hard to measure directly. As such, SMEs are traditionally identified using proxies of expertise such as experience, job performance, professional credentials, or peer recommendations (Bergman, Drasgow, Donovan, Henning, \& Juraska, 2006).

These traditional methods of selecting SMEs have several drawbacks. Peer recommendations, for example, are often biased by popularity and familiarity, both of which are unrelated to performance (Shanteau et al., 2002). Moreover, meta-analytic evidence shows that tenure and experience are only weakly associated with overall job performance (Schmidt \& 
Hunter, 1998; Van Iddekinge et al., 2019). In addition, top performers may not themselves be aware of necessary skill sets to achieve high performance, especially when it involves social situations that are often highly variable and easily influenced by external factors. Indeed, research has shown that proxies such as tenure and job performance are inadequate for measuring the quality of judgments in complex social situations such as those in the workplace (Cucina et al., 2012). The absence of normative criteria for social judgments can lead to concerns of the predictability and interpretability of SJTs (see Campion et al., 2014, for review). Because of all these reasons, there is a need for an objective method of identifying subject matter expertise in the context of SJT development.

In this paper, we argue that traditional criteria such as experience and credential alone are not sufficient indices of expertise in complex social situations where a normative criterion may not be available. We introduce the Cochran-Weiss-Shanteau (CWS) approach—an empirically based theory of expertise — as a potentially viable method for identifying SMEs in such situations (Weiss et al., 2006). We first outline the characteristics of expert judgments and describe how the CWS approach may be used for identifying expert judgments. Next, we conducted two empirical studies to examine the utility of the CWS index and its construct validity. In the first study, we present an empirical demonstration using a college sample to illustrate how test creators may incorporate the CWS index in their work. In the second study, we examined the convergent and divergent validity of the CWS index. Finally, we discuss the advantages of the CWS approach and how it can be used to measure expertise in other employee selection domains.

\section{An Empirical Theory of Expert Judgment}

What constitutes an expert judgment? We have mentioned above a few traditional methods such as domain performance (e.g., job performance in the work domain), professional 
accreditation, and peer approval. These traditional methods, however, are likely to be heavily influenced by subjective judgments and biases that are unrelated to one's actual performance. Besides these methods, another traditional method to determine expert judgment is its correspondence with normative correctness. The expertise of a mathematician, for example, is based on whether they are able to produce objectively normatively correct solutions to math problems. Of course, this approach assumes that a normative correct solution exists a priori. Unlike math problems, many social and work situations do not necessarily have an objectively correct solution. For example, deciding whether it is appropriate to give a discount against company policies may be 'correct' in some situations, but not others. An employee's judgment will also depend on personal motivation as well as unique organizational constraints. In these situations, then, how does one determine the expertise of human judgment?

In this paper, we introduce a theory of expertise that does not rely on external validation (normative correctness, domain performance) or approval (accreditation, peer approval). This approach deviates from the philosophies of traditional psychometric theory in the treatment of the 'ground truth'. On a test of cognitive ability, for example, the correctness of an answer corresponds to a certain level of the trait. Even non-cognitive measures (e.g., personality) contain answers that corresponds to high standing on a trait. This approach is sensible in situations where ground truth (i.e., correct answer) exists, or is assumed to exist. In complex social situations where human judges are required, we contend that ground truth is harder, or even impossible, to ascertain. For instance, an appropriate response to a social dilemma at work often requires considerations of unique contextual factors such as job requirements, organizational norms, or departmental politics. We argue that in such situations, the concept of expertise becomes relative, rather than absolute (Shanteau et al., 2002). Like judging the quality of fine wine, best judgment 
in one work situation for one employee may not necessarily be the best for another. The assessment of expertise in these situations, therefore, calls for an approach that does not rely on the presence of a normative criterion.

Unlike traditional psychometric theories, the empirical approach suspends the assumption that a "ground truth" exists. Consider the expertise of wine tasters. Based on a traditional approach, the expertise of a wine taster is determined based on the frequency by which they 'correctly' identify objectively 'good' wines. This approach requires a determination, a priori, of what constitutes as a 'good' bottle of wine. This approach is not feasible if there is not a normative criterion for 'good' versus 'bad' wine. In comparison, using the empirical approach, wine tasters may be asked to taste different glasses of wine, each more than once. The expertise of a wine taster can be determined based on if they produce different quality judgments when tasting different wines and if it produces consistent judgments when tasting the same wine multiple times. An empirical approach does not require the presence of a normative criterion in terms of the wine's objective quality to validate the taster's judgments. This empirically based approach, known as the Cochran-Weiss-Shanteau (CWS) approach (Weiss et al., 2006), serves as the basis of assessing expertise of human judges in domains where expertise may be relative and normative criterion may be hard to ascertain.

Based on the CWS approach, expertise can be ascertained based on the empirical characteristics of the judgments alone (Einhorn, 1974), without correspondence to any external validation. From the empirical perspective, a person's expertise can be partially determined based on whether they make consistent judgments toward the same stimuli over time. For example, an expert judge of social and work situations should give consistent responses to the same situation at multiple test administrations. This method is particularly useful for judging the 
quality of complex social judgments that, like judging fine wine, lack a normative criterion for correctness (Shanteau et al., 2002). Consistency, therefore, may serve as an index of expert judgment when there are no objective criteria (Bolger \& Wright, 1992; Einhorn, 1974).

Consistency alone, however, is not enough to merit expert judgment because it can arise from giving all stimuli the same response without discriminating among different stimuli. For example, a manager could judge every job candidate as qualified when some are not; a test taker can respond to every multiple-choice question with B even though many of them are incorrect. Both cases are examples of consistent, but poor overall judgments because the judge ignored true differences in the choices. Therefore, in addition to consistency, there should be a second criterion for expert judgment: discriminability, or the capability of discriminating between true differences across stimuli. Hammond (1996) argued that experts should be able to make subtle distinctions between different stimuli by attending to variations in relevant cues (also see, Abdolmohammadi \& Shanteau, 1992). Both consistency and discriminability, therefore, are essential characteristics of expert judgments. A good hiring manager, for example, should give different ratings to two differentially qualified candidates and consistent ratings to candidates with the same or similar qualifications. In sum, expert judgment should be both highly consistent and discriminable, and this premise forms the basis of the CWS approach of expert judgment (Weiss \& Shanteau, 2003).

The CWS approach of expertise incorporates discriminability (when judging different stimuli) and consistency (when judging the same stimuli) as behavioral indices of expert judgment. The CWS Index is a single numerical value that reflects a respondent's expertise based on a set of empirical judgments. Specifically, it takes the ratio of discrimination and inconsistency. Discrimination is the respondent's variability in his or her evaluations across 
different stimuli; inconsistency is the variability of his or her evaluations of the same stimuli over time.

The CWS approach has been used as a measure of expertise in a variety of domains such as clinical diagnoses, hiring decisions, and weather forecasts (Pauley et al., 2009; Shanteau et al., 2002; Williams et al., 2008). Pauley et al. (2009) used the CWS index as a measure of expertise in aeronautical risk judgments, where good judgment is essential for identifying good pilots. At the same time, the level of risk in any given situation is uncertain and subject to various contextual and circumstantial factors. Using a realistic task involving weather-related risks, they found that the CWS index served as a valid measure of expertise amongst aircraft pilots. The CWS index has also been used in clinical judgment contexts. Witteman et al. (2012), for example, successfully used the CWS index to estimate the expertise of counselors for assessment of depressive disorder. These examples demonstrate the utility of the CWS index for identifying expert judges in situations where there is considerable uncertainty in the normative criterion and human judges are required.

\section{CWS Approach for Identifying SMEs}

As we mentioned above, when identifying SMEs for an SJT, it is important to assess the actual expertise of potential SMEs rather than relying on other proxies such as job performance or experience. We argue that the CWS index, given its advantages in assessing expertise, especially in situations where it is hard to determine normative criteria (e.g., social situations at work), could be applied to the work context to assess the expertise of potential SMEs. Below, we describe how the CWS approach may be used to identify potential SMEs in the context of SJT development. 
Step 1) Administering the SJT. To obtain the CWS index, the potential pool of SMEs would first take a uniform set of SJT items drawn from the pool of possible items. In practice, the CWS index can be calculated with as few as five unique judgments (e.g., items) (Weiss \& Shanteau, 2014). Discriminability is calculated based on the variance of the respondents' answers across different situations (test items). Consistency is calculated based on the variance of responses to the same item which means the questions will have to be repeated to the respondents. As such, to modify a standard SJT to cooperate with the CWS approach, one can repeatedly administer a random subset of the SJT items within the same assessment. Admittedly, respondents may try to recall their previous answer rather than making an independent judgment, which may distort the ability of this approach to assess the respondents' judgment ability accurately. As such, the memory effects may be minimized by introducing some time lag or cognitive redirection before introducing the repeated items.

Step 2) Calculating the CWS Index. The CWS approach accommodates calculating a CWS index based on either interval (e.g., Likert scale) or nominal (e.g., multiple choice) response scales. For interval or ordinal data, the CWS index can be calculated as the ratio between the variance in responses to different stimuli (discriminability) and variance in responses to the same stimuli across administrations (inconsistency). The resulting ratio reflects a tradeoff between discrimination across items and (in)consistency within items.

For nominal or categorical data, a non-parametric procedure is required. Appendix A provides a hypothetical example of calculating the CWS index with nominal responses, which we illustrate here because SJTs typically use multiple choice questions that produce nominal responses. The $R$ code for calculating a person's CWS index from nominal data can be found on 
the author's Github' ${ }^{1}$ With nominal data, consistency and discriminability are both measured by the degree to which responses match (e.g., rater A chooses the same option for both times) or mismatch (e.g., rater A choose option B for Time 1 but option C for Time 2). In order to obtain the CWS index, one needs to first calculate inconsistency (the reverse indicator for consistency) by the ratio of the number of mismatches within the same item over the total number of possible within-item matches; one also needs to calculate discrimination with the ratio of the number of mismatches across different items over total possible across-item matches. CWS index is then calculated by putting the discrimination score as the numerator, divided by the inconsistency score as the denominator.

Step 3) Interpreting the CWS Index. The CWS index reflects the ratio between discrimination and consistency. A high CWS index would indicate judgments that are highly discriminable and consistent (i.e., low in inconsistency), both of which necessitate expert judgments. Note that the CWS index is not a standardized metric; the value of this index varies depending on factors such as the stimuli and scale used to measure judgments. For example, a test with a disproportionate number of B as the correct answer may affect the interpretation of results. Therefore, the CWS indices can only be meaningfully compared between individuals or groups when their CWS indices are calculated based on the same set of items and judgments.

\section{Study 1: Demonstration with the College Situational Judgment Inventory}

The purposes of the first study are three-fold. First, we demonstrate how the CWS index may be used to identify subject matter experts. Second, using CWS index as an indicator, we compare the expertise of lower and advanced level students. Specifically, we examine if there is

\footnotetext{
${ }^{1}$ https://github.com/dczhang1/cwsNominal
} 
a difference in CWS scores between the two groups of students. And third, we compare the CWS method with the rational approach by assessing the interrater agreement of the SMEs identified with each method. Specifically, we examine if expert judges — as a group — would produce greater interrater agreement than non-experts and if the difference in interrater agreement between groups depended on the mode of SME selection (tenure-based vs. CWS-based).

We used the college situational judgment inventory for the demonstration (SJI, Oswald, Schmitt, Kim, Ramsay, and Gillespie, 2004). The SJI consist of 57 items that cover the 12 dimensions of college performance categorized into three broad dimensions: intellectual, interpersonal, and intrapersonal (See Appendix B, for a sample of items). In the present study, we used these three overarching dimensions to create three versions of SJI to administer to participants. The original authors used a rational approach to develop the scoring key and selected their subject matter experts based on seniority (juniors and senior college students; Oswald et al., 2005). SMEs first compiled critical incidents, and then they were asked to indicate what they judge to be the best/worst responses for each scenario. SME's input is used to form the scoring key for the SJI. In the original development of the SJI, items with less than $75 \%$ agreement amongst the 42 SMEs were discarded from the item pool.

\section{Study 1: Method}

\section{Sample}

We obtained data from 102 undergraduate psychology majors at two large public universities in the United States. Due to the length of the SJI, each participant only completed one of three versions of the SJI to reduce participant fatigue. Each version corresponds to one of the three major dimensions on the SJI (intellectual, intrapersonal, and interpersonal). And each form has several specific sub-dimensions (e.g., perseverance, knowledge, etc). We removed 29 
total participants who missed more than one out of three possible attention check question where we embedded — in the item description — the statement "if you are still paying attention, please select A as the best option for this question." The final sample $(\mathrm{n}=73)$ was $95 \%$ female $(40 \%$ freshman, $21 \%$ sophomore, $25 \%$ junior, $15 \%$ senior).

\section{Procedure}

We administered the SJI twice to our sampled students, with a two-month lag between time points. To minimize response fatigue, each participant responded to one of the randomly chosen three forms (Interpersonal, $n=19$, Intrapersonal, $n=28$, Intellectual, $n=26$ ) of the SJI. At Time 1, all participants completed the assigned version of the SJI in its entirety. At Time 2, all participants completed a shortened SJI, which is a subset (roughly $25 \%)^{2}$ of the items from Time 1. The repeated subset of items is used to calculate the CWS index based on the nominal approach (Weiss \& Shanteau, 2005).

\section{Study 1: Results}

Seniority-based expertise was separated into upperclassmen (junior and seniors) and underclassmen (freshmen). We limited the tenure-based novice group to freshmen to maximize the power to observe any true difference in students' answers due to experience. The study was conducted during the fall semester, which allowed us to observe how freshmen without college experience would respond to our SJT measures, and whether these responses were different from those with two or more years of college experience. We chose not to include sophomores in the

${ }^{2}$ Before the study, we selected, randomly, one item from each sub-dimension for each of the three SJI forms. Due to the different number of dimensions in each of the three SJI versions, the final number of repeated items for the intellect, interpersonal, and intrapersonal forms were three, four, and five, respectively. 
group of novices because they have an additional full year of experience, and thus, do not constitutes as true novices in a college setting.

We did not find a significant difference in CWS index between upper (juniors and seniors) and underclassmen (freshman) for any of the three SJI dimensions (Table 1) ${ }^{3}$. We did observe a pattern of difference such that underclassmen appear to have slightly, though nonsignificant, higher CWS scores than upperclassmen ${ }^{4}$. The very small and nonsignificant differences between under- and upperclassmen are likely due to chance.

Next, we compared the traditional approach (expertise based on seniority) to the CWS approach (expertise based on CWS index) in identifying "experts" and "non-expert" judges. Under the traditional approach, first-year undergraduate students were categorized as nonexperts, and third/fourth-year students were identified as experts. Under the CWS approach, we categorized experts and non-experts based on a median split such that students with a CWS index above the median were categorized as experts, and vice versa. We also included comparisons between the top quartile vs. bottom quartile in CWS index.

We compared the CWS approach to traditional (experience-based) approach for identifying judges in terms differences in interrater agreement. We calculated various indices for interrater agreement using participants responses to all the SJT items at Time 1. For each group of expert and non-expert judges; we calculated the average percent of interrater agreement for each item in the SJI as well as the mean of percent agreement across all items (Table 2). Mean

${ }^{3}$ We calculated CWS scores for underclassmen to include sophomores. The resulting CWS scores were 2.39, 2.15, and 2.17 for Intellect, Interpersonal, and Intrapersonal respectively. Results for the difference in CWS index between upper vs. underclassmen were unchanged. 
percent agreement for each item is calculated by taking the percent of participants responding the most frequently endorsed option. Results based on the traditional approach showed that there was minimal difference in percent agreement between under- and upperclassmen for the intrapersonal and intellectual SJI versions. Interestingly, upperclassmen had a slightly higher agreement in the interpersonal dimension than underclassmen. Based on the CWS approach, we found that expert judges had a greater percent of agreement than non-expert judges in both the interpersonal and intellectual dimensions.

We also calculated Fleiss' Kappa with 95\% confidence interval (95\% CI) as an index for inter-rater agreement using the raters package in $R$ (Giardiello et al., 2015). We examined the degree of overlap in the $95 \%$ CIs of Kappa estimates between experts and non-experts. We found minimal differences in 95\% CIs Kappa between upperclassmen and underclassmen for the interpersonal and intellectual dimensions. We did find that upperclassmen judges produced a slightly higher, but non-overlapping, Kappa than underclassmen for the intrapersonal dimension. The 95\% CIs Kappas did not overlap between CWS-based expert vs. nonexperts for all three dimensions. More importantly, the Fleiss' Kappa for CWS-based experts was higher than tenurebased experts in two of the three dimensions (interpersonal and intellectual).

\section{Study 2: Construct validity of CWS index}

The first study demonstrated how the CWS index may be used in a SJT context. The underlying construct reflected by the CWS index, however, remains unclear. In the second study, we explore this issue by examining three competing theoretical mechanisms underlying the CWS index.

From a theoretical perspective, it is unclear whether the CWS index reflects a situationspecific aspect of expertise or a general capacity to make good judgments in any situation 
(Lievens \& Motowidlo, 2016). There are reasons for both perspectives. Considering that SJTs are usually developed based on a specific psychological construct (e.g., interpersonal skills; integrity), the ability to make consistent and discriminatory judgments in situations depicted in the SJT could reflect a person's standing on the construct relevant to the SJT. The CWS index obtained from a SJT of integrity, for example, should reflect one's standing on integrity. Based on the domain-specific perspective, respondents' performance on the CWS index during a SJT is predicted by their standing on the latent trait associated with the SJT.

There are also reasons that the CWS index ascertained from SJT responses reflects a domain-general capacity to make good judgments (Brooks \& Highhouse, 2006), which differs from the latent construct associated with the SJT. This perspective mirrors a growing body of research suggesting that SJTs are better characterized as measures of domain-general (i.e., context-free), rather than situation-specific knowledge and skills (Jackson et al., 2017; Lievens \& Motowidlo, 2016; Schäpers et al., 2020). This research has shown, for example, removing the situation description from SJTs makes little difference to the validity SJTs (Krumm et al., 2015). High performers on the SJT relied on domain-general knowledge and principles of good judgements. Taking this approach, we assert that expert judgment, as operationalized by the CWS index, reflects one's ability to exercise reflective thinking in ambiguous situations by applying the same rationale and reasoning when making decisions across situations.

Reflective thinking allows the judge to deliberately evaluate situations and arrive at a judgment based on rational thinking, rather than gut feelings (Epstein et al., 1996; Frederick, 2005). As a result, their judgments are more discriminatory because they are better able to discern different situations and more cross-temporally consistent, which reduces the inconsistency. Based on a domain-general perspective, expert judgment does not necessarily 
require the judge to have high standing on the latent trait related to the situations per se. Instead, we posit that the expert judgment should be positively associated with reflective thinking, and negatively associated with decision making styles, such as rational and intuitive decision making. We also posit that CWS index may also be related to how people think and process information in general. For instance, those who tend to think reflectively may score high on the CWS index.

In addition to the two theoretical perspectives, we also considered a third, methodological explanation for the CWS index. Considering that a key component of the CWS index is the consistency in which people make judgments toward the same stimuli across multiple administrations, it is possible that the CWS index performance simply reflects careless responding (i.e., test motivation). Indeed, one behavioral index of careless responding considers the consistency of responding to multiple items of the same content in a survey. Participants who respond to the same item very differently, for example, are marked as careless (Huang et al., 2015). It is worth mentioning, however, that the methodology for identifying careless responders examines item responses in the same administration. When there is a time gap between item responses, however, it becomes more tenuous to argue that inconsistent responses across time necessarily reflects carelessness. Still, we test the plausibility of the methodological explanation for the CWS index.

To empirically test the three competing explanations for the CWS index, we conducted a time-separated survey study with working adults. Because we aimed to gather data from employees in a variety of occupations, we used a SJT of employee integrity. We chose this SJT because the like many social situations, the 'correct' response to integrity-related situations can be subjective, which highlights the importance of adopting the CWS index. Like Study 1, we 
administered the identical set of SJT items across two time periods in order to calculate the CWS index.

We included a number of measures to test the competing hypotheses. First, we included a construct-based measure of integrity to test the domain-specific hypothesis that CWS index reflects expertise in the domain associated with the SJT. Next, we included two measures related to reflective thinking and decision styles. The first is the Cognitive Reflection Test, which is a performance-based measure of cognitive reflection, and the second is a self-report measure of decision-making style that captures both rational and intuitive decision styles. Finally, we included several attention-check questions as a behavioral measure of carelessness. We also included a self-report measure of conscientiousness as a dispositional measure of survey motivation (Bowling et al., 2016). For exploratory purposes, we also examined subjective career success as an outcome.

\section{Study 2: Method}

\section{Sample and Procedure}

We used a time-separated panel survey design. We gathered data from 323 employees $(71 \%$ female, mean age $=35[S D=11.4], 78 \%$ Caucasian $)$ using Prolific ${ }^{5}$, which is an online crowdsourcing platform used for recruiting a diverse sample of participants for research in the social sciences. Prolific also allows researchers to pre-screen for demographic characteristics such as occupational status and job-seeking motives. For this study, we only included respondents who are currently employed full time. Individual difference measures, demographic information and the SJT were administered at Time 1. Approximately 4 weeks later, we

\footnotetext{
${ }^{5}$ www.prolific.ac
} 
administered the second wave of the survey, which contained the same set of SJT items as those administered at Time 1 as well as a measure of subjective career success. The response rate of the Time 2 survey was $81 \%$.

We included three attention check questions throughout the two surveys. First, we asked an open-ended question where respondents were asked to indicate the last task they performed on the job. We manually reviewed each response to look for non-sense answers and indication of potential bots. The second attention check item was a survey question where participants indicated whether they agreed with the statement: "I do not read or speak English". The third attention check item was embedded in one of the SJT items where the participants were instructed on how to respond. An inattentive score was calculated based on the number of attention check questions that the participants missed. Twenty-eight participants missed one or more attention check questions.

\section{Measures}

CWS Index. We used four items chosen from a SJT of employee integrity developed by Becker (2005) to calculate the CWS index. We chose items that contained work situations that we felt applied to most occupations (see Appendix C, for a list of items). As we noted before, the CWS index can be calculated with only a few item responses as long as the same items are administered multiple times. Each SJT item contained five nominal options. Participants were asked to indicate what they are most and least likely to do for each item. In total, participants gave eight responses to four SJT items at Time 1 and again at Time 2. The CWS index was calculated based on the discriminatory and inconsistency of the participants' sixteen total judgments over two time periods. Due to the heavy-tail distribution of the CWS index, we transformed the scores using log transformation. 
Cognitive Reflection Test. We used the CRT-2 (Thomson \& Oppenheimer, 2016), a revised test of cognitive reflection. The test contains logic-based questions that have an intuitive but incorrect answer, which could be avoided with more reflection. One example item is " $\mathrm{A}$ farmer had 15 sheep and all but 8 died. How many are left?’. The intuitive but incorrect answer to this question is 7. Upon further reflection, however, one should ascertain that there are actually 8 sheep left. The CRT score is calculated based on the total number of correct responses and has an internal consistency of .64.

Decision Style. We used the 10-item measure of rational-intuitive decision style developed by Hamilton et al. (2016). It measures decision style along two orthogonal dimensions: intuitive style ("I make decisions based on intuition”) and rational style ("I weight a number of different factors when making decisions"). The internal consistencies for the two subscales were .83 and .84 respectively.

Integrity. We used a personality-based measure of integrity developed by Catano et al. (2018). This measure conceptualizes integrity as a second-order personality construct comprised of agreeable, conscientiousness, and emotional stability. Accordingly, it contains 10 items that reflect these three personality traits. An example item is "I always treat others with kindness". The internal consistency was .78.

Conscientiousness. We used the conscientiousness dimension of the IPIP-NEO60 (Maples-Keller et al., 2019), which contained 12 self-report items measuring the six facets of conscientiousness as a big five personality trait. The internal consistency was .77.

Subjective Career Success. We used 24-item measure of subjective career success developed by Shockley et al. (2016). It measures one's own evaluation of their career success 
eight dimensions (e.g., influence, quality of work, authenticity). The internal consistency was .92.

\section{Study 2: Results}

We first examined whether CWS index reflects careless responding. To do so, we examined the bivariate correlation between attentiveness score and CWS index for the full sample. Although Pearson's correlation revealed a negative but non-significant association $\left(r_{\text {pearson }}=-.07, p=.25\right)$; a rank-order correlation (i.e., Spearman's correlation) revealed a marginally significant negative association $\left(r_{\text {spearman }}=-.12, p=.05\right)$. Together, the careless aspect of the CWS index is negligible.

We tested the nomological network of the CWS index using only the attentive participants, which methodologically controls for the attentiveness aspect of the CWS index performance. Table 3 contains the bivariate correlations between the study's variables. The CWS index was positively correlated with cognitive reflection $(r=.17, p=.008)$, and negatively correlated with intuitive decision style $(r=-.14, p=.02)$. It was not, however, associated with work experience, integrity, rational decision style, conscientiousness, nor subjective career success.

\section{General Discussion}

Despite the prevalence of the rational scoring method in SJTs and the reliance on SMEs, very little research is devoted to understanding the characteristics of the SMEs and the quality of their judgments. Scoring keys for the SJT are usually based on the characteristics of the item, with little regard for the characteristics of the judges. Our paper describes an empirical approach to understand expert judgments: one based on the behaviors of the judges rather than their 
experience or credentials. We found that SMEs chosen based on high CWS index had greater interrater agreement, which is important for the efficient development of scoring keys.

In both studies, we found that the CWS index was not associated with work or school experience. Our findings are consistent with past research on the CWS index that fails to find a significant association with domain experience (e.g., Pauley et al., 2009; Witteman et al., 2012). Together, these findings suggest that CWS index may not reflect job-specific or domain-specific knowledge that can be acquired through experience. In the second study, we found the CWS index calculated based on an integrity SJT was associated with individual differences in cognitive reflection and intuition-avoidance, rather than integrity. These findings suggest that the CWS index also may not reflect the latent construct related to the test itself (e.g., integrity). Instead, our results suggest that CWS index appears to reflect a domain-general capacity to make good judgments, which is associated with the tendency to exercise deliberative thinking and decision-making: people who tend to make intuitive decisions also tend to have lower scores on CWS index whereas people who engage in more reflection tend to have higher scores on the CWS index.

\section{Theoretical Implications}

The conceptualization of the CWS index as a context-free capacity to make good judgments rather than job-specific knowledge has several theoretical implications for SJT research. Our findings suggest that in addition to traditionally studied antecedents of SJT performance (e.g., job experience, cognitive ability, and personality), individual differences in deliberation, as reflected by cognitive reflection, may also affect performance on the SJT. One explanation for our findings is that individuals who exercise cognitive reflection are more consistent in their situational construal such that they interpret situational cues given in SJT 
items more consistently (Melchers \& Kleinmann, 2016). In contrast, participants who engage in more intuition-based thinking may be interpreting vague situational cues in SJT items differently across administrations, thereby resulting in lower scores on the CWS index due to greater inconsistency. We did not find that the CWS index calculated from an integrity SJT was associated with the integrity construct. This suggests that the CWS index may serve as a totally context-free measure of SJT performance. Our approach complements with existing methods of studying the situational influences of SJTs. Whereas traditional methods have focused on how varying situational cues in SJT descriptions affect test validity, our approach (the CWS approach) shifts the focus to the criterion. By introducing a context-free measure of SJT performance, we offer SJT scholars another tool to study how people respond to SJTs and the degree to which SJT captures situation-specific knowledge or domain-general ability.

\section{Practical Implications}

Organizations often call upon practitioners to develop SJTs tailored to their unique needs. We believe the CWS-approach could complement traditional approaches to identify SMEs when developing answer keys for SJT, because it provides a behavioral index of expertise for situations where "correct answers" may be ambiguous or not practical to obtain, which is often the case for SJT development. Specifically, practitioners may use the CWS approach to evaluate the pool of potential SMEs in a multiple-hurdle process. For example, after individuals are identified based on a traditional method such as credentials or experience, it may be useful — if feasible - to administer a small sampling of potential judgments as a way to ascertain the CWS index of the pool of potential judges and eliminate the poor performers. As shown in our study, the inclusion of SMEs with low CWS-score may introduce more disagreement in the judgments, thereby slowing down the key-generation process. The CWS index can also be used for developmental 
purposes. Experienced SMEs who performed poorly could be placed in specific training programs to improve their performance. Some evidence suggests expert judgment can be trained. Weiss, Shanteau, and Harries (2006) used the CWS approach in a training study of occupational therapists. They found that judgment quality, as measured with the CWS approach, improved after several 1-hour training sessions. One potential intervention is to encourage judges to explicitly reflect on their decision rationale and rely less on their gut feelings. Doing so may be beneficial for the efficient development of SJTs.

\section{Limitation and Future Directions}

Therefore, future research should further examine the domain-generalizability of judgment expertise in SJTs using the CWS index. Specifically, more research is needed to examine the degree to which CWS index generalizes across items and SJTs. Although we have shown that the CWS index may result in a more efficient key generation process (e.g., better interrater agreement), it is uncertain if the resulting scoring key would also improve the construct and predictive validity of the SJT (e.g., Bergman et al., 2006). Therefore, future research should examine whether the scoring keys generated by CWS-based experts differ from those generated by experience-based experts, and how the differences affect the validity of the SJTs. Relatedly, more research is needed to understand why judges with high CWS scores produce higher interrater agreement. It may therefore be helpful to accompany the CWS index with in-depth qualitative evidence, such as focus-group interviews with the identified experts, to further examine the quality of the judgments and decisions. Considering that the CWS index is calculated based on response patterns, it is likely to be influenced by other basic cognitive processes such as attention and memory. Although we did not find survey attentiveness to be a strong predictor of the CWS index in this sample, this finding may be hindered by the restricted 
range in respondent attentiveness in our sample. More research is needed to understand how cognitive processes of survey response affect the performance on the CWS index.

\section{Conclusion}

Subject matter expertise can be hard to ascertain in situations where 'good judgment' is subjective. Yet, the selection of subject matter experts is necessary for the development of situational judgment tests. In this paper, we introduce an empirically based theory of expertise: the Cochran-Shanteau-Weiss (CWS) approach, which does not rely on the presence of normative criterion, as a method of selecting SMEs that may be combined with existing indices such as job experience and credentials. Our studies illustrated how the CWS index may be used to identify candidate SMEs and that score on the CWS index appears to be driven by reflective thinking and an avoidance toward using intuition when making decisions. 


\section{References}

Abdolmohammadi, M. J., \& Shanteau, J. (1992). Personal attributes of expert auditors. Organizational Behavior and Human Decision Processes, 53(2), 158-172.

Becker, T. E. (2005). Development and validation of a situational judgment test of employee integrity. International Journal of Selection and Assessment, 13(3), 225-232.

Bergman, M. E., Drasgow, F., Donovan, M. A., Henning, J. B., \& Juraska, S. E. (2006). Scoring situational judgment tests: Once you get the data, your troubles begin. International Journal of Selection and Assessment, 14(3), 223-235.

Bolger, F., \& Wright, G. (1992). Reliability and validity in expert judgment. In Expertise and decision support (pp. 47-76). Springer. http://link.springer.com/chapter/10.1007/978-0585-34290-0_4

Bowling, N. A., Huang, J. L., Bragg, C. B., Khazon, S., Liu, M., \& Blackmore, C. E. (2016). Who cares and who is careless? Insufficient effort responding as a reflection of respondent personality. Journal of Personality and Social Psychology, 111(2), 218.

Brooks, M. E., \& Highhouse, S. (2006). Can good judgment be measured. Situational Judgment Tests: Theory, Measurement, and Application, 39-56.

Campion, M. C., Ployhart, R. E., \& MacKenzie Jr, W. I. (2014). The state of research on situational judgment tests: A content analysis and directions for future research. Human Performance, 27(4), 283-310.

Catano, V. M., O’Keefe, D. F., Francis, R. E., \& Owens, S. M. (2018). Construct-based approach to developing a short, personality-based measure of integrity. International Journal of Selection and Assessment, 26(1), 75-92. 
Christian, M. S., Edwards, B. D., \& Bradley, J. C. (2010). Situational Judgment Tests: Constructs Assessed and a Meta-Analysis of Their Criterion-Related Validities. Personnel Psychology, 63(1), 83-117. https://doi.org/10.1111/j.1744-6570.2009.01163.x

Cucina, J. M., Caputo, P. M., Thibodeaux, H. F., \& Maclane, C. N. (2012). UNLOCKING THE KEY TO BIODATA SCORING: A COMPARISON OF EMPIRICAL, RATIONAL, AND HYBRID APPROACHES AT DIFFERENT SAMPLE SIZES: JEFFREY M. CUCINA ET AL. Personnel Psychology, 65(2), 385-428. https://doi.org/10.1111/j.1744$6570.2012 .01244 . \mathrm{x}$

Einhorn, H. J. (1974). Expert judgment: Some necessary conditions and an example. Journal of Applied Psychology, 59(5), 562.

Epstein, S., Pacini, R., Denes-Raj, V., \& Heier, H. (1996). Individual differences in intuitiveexperiential and analytical-rational thinking styles. Journal of Personality and Social Psychology, 71(2), 390.

Frederick, S. (2005). Cognitive reflection and decision making. The Journal of Economic Perspectives, 19(4), 25-42.

Giardiello, D., Quatto, P., Ripamonti, E., \& Vigliani, S. (2015). “raters”, A Modification of Fleiss' Kappa in Case of Nominal and Ordinal Variables (2.0.1) [R].

Hamilton, K., Shih, S.-I., \& Mohammed, S. (2016). The Development and Validation of the Rational and Intuitive Decision Styles Scale. Journal of Personality Assessment, 1-13.

Hammond, K. R. (1996). Human judgement and social policy: Irreducible uncertainty, inevitable error, unavoidable injustice. Oxford University Press. http://psycnet.apa.org/psycinfo/1996-98949-000 
Huang, J. L., Liu, M., \& Bowling, N. A. (2015). Insufficient effort responding: Examining an insidious confound in survey data. Journal of Applied Psychology, 100(3), 828.

Jackson, D. J., LoPilato, A. C., Hughes, D., Guenole, N., \& Shalfrooshan, A. (2017). The internal structure of situational judgement tests reflects candidate main effects: Not dimensions or situations. Journal of Occupational and Organizational Psychology, 90(1), 1-27.

Krumm, S., Lievens, F., Hüffmeier, J., Lipnevich, A. A., Bendels, H., \& Hertel, G. (2015). How "situational" is judgment in situational judgment tests? Journal of Applied Psychology, 100(2), 399-416. https://doi.org/10.1037/a0037674

Lievens, F., \& Motowidlo, S. J. (2016). Situational judgment tests: From measures of situational judgment to measures of general domain knowledge. Industrial and Organizational Psychology, 9(1), 3-22.

Lievens, F., Peeters, H., \& Schollaert, E. (2008). Situational judgment tests: A review of recent research. Personnel Review, 37(4), 426-441.

Maples-Keller, J. L., Williamson, R. L., Sleep, C. E., Carter, N. T., Campbell, W. K., \& Miller, J. D. (2019). Using Item Response Theory to Develop a 60-Item Representation of the NEO PI-R Using the International Personality Item Pool: Development of the IPIP-NEO-60. Journal of Personality Assessment, 101(1), 4-15. https://doi.org/10.1080/00223891.2017.1381968

Melchers, K. G., \& Kleinmann, M. (2016). Why situational judgment is a missing component in the theory of SJTs. Industrial and Organizational Psychology, 9(1), 29.

Oswald, F. L., Schmitt, N., Kim, B. H., Ramsay, L. J., \& Gillespie, M. A. (2004). Developing a Biodata Measure and Situational Judgment Inventory as Predictors of College Student 
Performance. Journal of Applied Psychology, 89(2), 187-207.

https://doi.org/10.1037/0021-9010.89.2.187

Pauley, K., O’Hare, D., \& Wiggins, M. (2009). Measuring expertise in weather-related aeronautical risk perception: The validity of the Cochran-Weiss-Shanteau (CWS) Index. The International Journal of Aviation Psychology, 19(3), 201-216.

Schäpers, P., Mussel, P., Lievens, F., König, C. J., Freudenstein, J.-P., \& Krumm, S. (2020). The role of Situations in Situational Judgment Tests: Effects on construct saturation, predictive validity, and applicant perceptions. Journal of Applied Psychology, 105(8), 800-818. https://doi.org/10.1037/ap10000457

Schmidt, F., \& Hunter, J. (1998). The validity and utility of selection methods in personnel psychology: Practical and theoretical implications of 85 years of research findings. Psychological Bulletin, 124(2), 262-274.

Shanteau, J., Weiss, D. J., Thomas, R. P., \& Pounds, J. C. (2002). Performance-based assessment of expertise: How to decide if someone is an expert or not. European Journal of Operational Research, 136(2), 253-263.

Shockley, K. M., Ureksoy, H., Rodopman, O. B., Poteat, L. F., \& Dullaghan, T. R. (2016). Development of a new scale to measure subjective career success: A mixed-methods study. Journal of Organizational Behavior, 37(1), 128-153. https://doi.org/10.1002/job.2046

Thomson, K. S., \& Oppenheimer, D. M. (2016). Investigating an alternate form of the cognitive reflection test. Judgment and Decision Making, 11(1), 99. 
Van Iddekinge, C. H., Arnold, J. D., Frieder, R. E., \& Roth, P. L. (2019). A meta-analysis of the criterion-related validity of prehire work experience. Personnel Psychology, 72(4), 571598.

Weiss, D. J., \& Shanteau, J. (2003). Empirical Assessment of Expertise. Human Factors: The Journal of the Human Factors and Ergonomics Society, 45(1), 104-116. https://doi.org/10.1518/hfes.45.1.104.27233

Weiss, D. J., \& Shanteau, J. (2005). CWS: A user's guide. Retrieved.

Weiss, D. J., Shanteau, J., \& Harries, P. (2006). People who judge people. Journal of Behavioral Decision Making, 19(5), 441-454. https://doi.org/10.1002/bdm.529

Whetzel, D. L., McDaniel, M. A., \& Nguyen, N. T. (2008). Subgroup differences in situational judgment test performance: A meta-analysis. Human Performance, 21(3), 291-309.

Williams, C. A., Haslam, R. A., \& Weiss, D. J. (2008). The Cochran-Weiss-Shanteau performance index as an indicator of upper limb risk assessment expertise. Ergonomics, 51(8), 1219-1237. https://doi.org/10.1080/00140130802087094

Witteman, C. L. M., Weiss, D. J., \& Metzmacher, M. (2012). Assessing Diagnostic Expertise of Counselors Using the Cochran-Weiss-Shanteau (CWS) Index. Journal of Counseling \& Development, 90(1), 30-34. https://doi.org/10.1111/j.1556-6676.2012.00005.x 
Table 1.

CWS Scores for Upper and Underclassmen

\begin{tabular}{lccc}
\hline SJI Dimension & Underclassmen & Upperclassmen & $t$-value \\
\hline Intrapersonal & 2.29 & 2.05 & 0.64 \\
Interpersonal & 2.18 & 1.93 & 0.58 \\
Intellect & 2.33 & 2.08 & 0.65 \\
\hline
\end{tabular}


Table 2.

Indices of interrater agreement for different groups of judges

\begin{tabular}{|c|c|c|c|c|c|c|}
\hline & \multicolumn{3}{|c|}{$\begin{array}{c}\text { Average \% } \\
\text { Rater Agreement } \\
\end{array}$} & \multicolumn{3}{|l|}{$\begin{array}{l}\text { Fleiss Kappa } \\
{[95 \% \text { CI }]}\end{array}$} \\
\hline & Inter. & Intra. & Intel. & Inter. & Intra. & Intel. \\
\hline \multicolumn{7}{|c|}{ Tenure-based approach } \\
\hline Upperclassmen & 0.68 & 0.59 & 0.64 & $0.34[.30, .38]$ & $0.30[.29, .32]$ & $0.33[.30, .35]$ \\
\hline Underclassmen & 0.62 & 0.58 & 0.62 & $0.32[.30, .34]$ & $0.25[.23, .28]$ & $0.33[.30, .35]$ \\
\hline \multicolumn{7}{|l|}{ CWS approach } \\
\hline Above Median & 0.67 & 0.59 & 0.68 & $0.38[.35, .40]$ & $0.30[.28, .31]$ & $0.42[.40, .44]$ \\
\hline Below Median & 0.59 & 0.56 & 0.58 & $0.27[.24, .29]$ & $0.25[.24, .27]$ & $0.26[.24, .28]$ \\
\hline Top Quartile & 0.73 & 0.59 & 0.68 & $0.48[.44, .53]$ & $0.27[.24, .30]$ & $0.43[.38, .45]$ \\
\hline Bottom Quartile & 0.61 & 0.54 & 0.58 & $0.27[.22, .31]$ & $0.17[.15, .20]$ & $0.25[.20, .29]$ \\
\hline
\end{tabular}

Notes. Inter. $=$ Interpersonal; Intra. $=$ Intrapersonal; Intel. $=$ Intellectual 
Table 3.

Means, standard deviations, and correlations of Study 2 variables

\begin{tabular}{|c|c|c|c|c|c|c|c|c|c|c|c|}
\hline Variable & $M$ & $S D$ & 1 & 2 & 3 & 4 & 5 & 6 & 7 & 8 & 9 \\
\hline 1. CWS Index & 0.86 & 0.61 & & & & & & & & & \\
\hline 2. Work Experience & 8.24 & 7.66 & .03 & & & & & & & & \\
\hline 3. Cognitive Reflection & 2.38 & 1.03 & $.17 * *$ & -.11 & & & & & & & \\
\hline 4. Rationality & 4.38 & 0.49 & -.07 & .08 & .06 & & & & & & \\
\hline 5. Intuition & 2.92 & 0.80 & $-.14^{*}$ & -.00 & $-.24 * *$ & -.07 & & & & & \\
\hline 6. Conscientiousness & 3.97 & 0.46 & .00 & $.25^{* *}$ & -.11 & $.26^{* *}$ & $-.19 * *$ & & & & \\
\hline 7. Integrity & 3.70 & 0.56 & -.02 & $.16^{*}$ & -.09 & $.16^{*}$ & -.04 & $.76^{* *}$ & & & \\
\hline 8. Career Success & 3.82 & 0.58 & -.05 & $.13^{*}$ & -.07 & .10 & -.00 & $.40 * *$ & $.30 * *$ & & \\
\hline 9. Sex & 1.76 & 0.43 & .11 & .06 & .00 & .00 & .07 & .02 & .01 & .05 & \\
\hline 10. Education & 4.30 & 1.53 & .04 & $-.20 * *$ & .11 & .09 & $-.16^{*}$ & .10 & .01 & $.19 * *$ & .02 \\
\hline
\end{tabular}

Note. $M$ and $S D$ are used to represent mean and standard deviation, respectively. $*$ indicates $p<.05 . * *$ indicates $p<.01$. Sex is coded as male $=1$; female $=2$. Education is coded as $1=$ less than high school; $2=$ high school; $3=$ some college; $4=$ Associate degree; $5=$ Bachelor'degree; $6=$ Master's degree; 7 = Doctoral degree; $8=$ Professional degree. 


\section{Appendix A. Calculating the CWS Index with Nominal Responses}

\begin{tabular}{lllll}
\hline & Item 1 & 2 & 3 & 4 \\
Time 1 & A & A & B & D \\
Time 2 & A & B & D & D \\
Match & 1 & 0 & 0 & 1 \\
\hline
\end{tabular}

\section{Computing possible within-column, across-column, and total matches.}

The total number of possible matches is calculated as 10 choose $2\left({ }_{10} \mathrm{C}_{2}=45\right)^{6}$ because there are 10 total responses and each response can match with one other response. Next, given there are 5 items ( 5 columns) and 2 responses per item, each item can have

\footnotetext{
6 The ${ }_{\mathrm{n}} \mathrm{C}_{\mathrm{k}}$ notation is a mathematical notation for Combinations. It is also written as $\left(\begin{array}{l}n \\ k\end{array}\right)$. Computationally, it can be derived with the formula $\frac{n !}{k ! \times(n-k) !}$. In combinatorics terms, Combination
} is the number of ways to choose a sample of $k$ elements from a set of $n$ distinct objects where order does not matter, and replacements are not allowed. In this example, with 10 responses, $10 \mathrm{C}_{2}$ derives the total number of ways to draw two unique elements (a pair of responses), regardless of whether the two items match or not. Therefore, the resulting value (45) is the total number of ways 10 responses can be paired. 
one possible match (same answers at Time 1 and Time 2). Therefore, there are $\underline{5}$ possible within-column matches. After removing the possible within-column matches, we are left with $\underline{40}$ possible across column matches.

\section{Computing non-matches within- and across-columns.}

Within-column non-matches can be computed simply by counting the number of mismatching response pairs within columns. In our example, there are two total within-column non-matches (e.g., items 2 and 3 each have one mismatching pair).

Across-column non-matches is conceptualized as the total pairs of discriminant responses across items. Arithmetically, this can be derived by subtracting the total number of across-columns matches from the total possible number of possible across-column matches (40).

Total number of across-column matches refers to the number of times that a response is repeated for a different stimulus. In our example, there are 2 across-column matches for the response option A (Item 1 (time 1) matches with Item 2 (time 1); Item 1 (time 2) matches with Item 2 (time 1)). Response option B had 1 across-column match: Item 2 (time 2) with Item 3 (time 1). And so on.

Algorithmically, one can also derive the total number of across-column matches by subtracting the across-column nonmatches from the total number of possible across-column matches. The exact details of the arithmetic is demonstrated in the $R$ script in Appendix B.

CWS numerator (discrimination) $=\sum \frac{\text { Nonmatches across columns }}{\text { Possible matches across columns }}=\sum \frac{40-(2+1+0+2)=35}{40}=0.875$ 
CWS denominator (inconsistency) $=\sum \frac{\text { Nonmatches within columns }}{\text { Possible matches within columns }}=\sum \frac{2}{5}=0.4$

CWS Index $\frac{0.875}{0.40}=2.19$ 


\section{Appendix B. Example Items of the SJI for Study 1}

\section{Knowledge}

Your grade for a particular class is based on three exams, with no class attendance requirement. All of the homework requirements for the class are posted on the professor's website. What would you do?

a) Attend class for as long as you feel that it is helping your grades

b) Do all the homework but only go to some of the lectures. It's the exams that count.

c) Go to all the classes anyway. The professor may say something important.

d) Skip classes, but if you did poorly on the first exam, start going to classes.

e) There is no need to go to classes. Just get the homework done, and pass the exams.

\section{Leadership}

An important class project you have been working on with a group of other students is not developing as it should because of petty differences and the need of some members to satisfy their own agenda. How would you proceed?

a) Try to solve the group problems before starting on the work.

b) Work hard by yourself to make sure the project is finished, taking on others' share of the work if necessary.

c) Talk to the professor and get suggestions about solving problems. If that doesn't work, try switching groups or have an independent project.

d) Schedule a number of meetings, forcing the group to interact.

e) Take charge and delegate tasks to each person. Make them responsible for their part of the project.

f) Talk to the group and demand that they start working together. 


\section{Appendix C. SJT Items for Study 2}

You are a food server in a busy restaurant. It's Friday night, and you have already put in 45 hours this week. The owner asks you to work tomorrow night, promising that you will be given a large dinner party which should get you a big tip. What are you most and least likely to say?

1. "I'd rather not, thanks.",

2. "Sure!"

3. "I'm kind of tired, but I'll do it if you can't find someone else."'

4. "I'll be happy to do it if you can guarante the tip."

You are a college professor, and two years ago you were given guaranteed job security. You've recently realized that this has decreased the quality of your work. What are you most and least likely to do?

1. Realize that this is natural - high job security leads to lower quality work.

2. Accept that you've moved to a new career stage where working so hard is not that important.

3. Admit that you've gotten a little lazy, set some high goals, and get back to being productive.

4. Find some things outside the workplace (e.g., hobbies, community work) that can replace the value you used to put on work.

LuAnn, one of your co-workers, comes to work wearing the ugliest blouse that you have ever seen. During a break she walks up to you and, after some small talk, asks, "How do you like my new blouse?' 'If you had to say one of the following, which would you mostly and least likely say?

1. "It's very pretty. Where did you get it?"

2. 'I don't think clothing is a very good measure of a person. Let's talk about you."

3. "Frankly, I don't find it too attractive."

4. "'You're asking the wrong person - I'm not a very good judge of clothes."

It is a beautiful day outside - sunny, warm, and inviting. You are scheduled to work, but you are tempted to take the day off and go to the beach with some friends. In all honesty, which of the following would you most and least likely do?

1. Call in sick and go to the beach.

2. Go into work and work as hard as you usually do.

3. Call your boss and tell him or her that you'd like to go to the beach, and see if your boss can find someone else to work for you.

4. Go into work but do not work as hard as normal. 